\title{
A SHARP BOUND FOR THE REGULARITY INDEX OF FAT POINTS IN GENERAL POSITION
}

\author{
M. V. CATALISANO, N. V. TRUNG, AND G. VALLA
}

(Communicated by Louis J. Ratliff, Jr.)

\begin{abstract}
A bound is given for the regularity index of the coordinate ring of a set of fat points in general position in $\mathbf{P}_{k}^{n}$. The bound is attained by points on a rational normal curve.
\end{abstract}

\section{INTRODUCTION}

Let $P_{1}, \ldots, P_{s}$ be distinct points in $\mathbf{P}_{k}^{n}, k$ an algebraically closed field, and let $m_{1}, \ldots, m_{s}$ be positive integers. If $\wp_{1}, \ldots, \wp_{s}$ are the prime ideals in $R:=k\left[X_{0}, \ldots, X_{n}\right]$ corresponding to the points $P_{1}, \ldots, P_{s}$, we let $Z:=$ $m_{1} P_{1}+\cdots+m_{s} P_{s}$ be the zero cycle defined by the ideal $\wp_{1}^{m_{1}} \cap \wp_{2}^{m_{2}} \cap \cdots \cap \wp_{s}^{m_{s}}$. If $m_{i} \geq 2$ the point $P_{i}$ is called a fat point of $Z$, a self-explanatory term. There is some interest in calculating the Hilbert function and the Betti numbers of the graded ring

$$
A=R /\left(\wp_{1}^{m_{1}} \cap \wp_{2}^{m_{2}} \cap \cdots \cap \wp_{s}^{m_{s}}\right),
$$

which is the homogeneous coordinate ring of $Z$.

It is well known that $A=\bigoplus_{t \geq 0} A_{t}$ is a one-dimensional Cohen-Macaulay graded ring whose multiplicity is $e:=\sum_{i=1}^{s}\left(\begin{array}{c}m_{i}+n-1 \\ n\end{array}\right)$, the degree of $Z$. This implies that the Hilbert function $H_{A}(t):=\operatorname{dim}_{k} A_{t}$ of $A$ is strictly increasing until it reaches the multiplicity, at which it stabilizes. The least integer $t$ for which $H_{A}(t)=e$ is called the regularity index of $A$ and denoted by $r(A)$. Note that since $A$ is Cohen-Macaulay, if $L$ is any linear form not vanishing at any of the points $P_{1}, \ldots, P_{s}$, then the artinian ring $B=A / L A$ has the property that $B_{t}=0$ iff $t>r(A)$. So, for an artinian ring $B$ we shall call its regularity the least integer $t$ such that $H_{B}(t)=0$.

Different results have been given on the postulation of the scheme $Z$ in the case of fat points (see [C, DG, C1, C2, G, G1, G2, H, S]), but they have been proved mostly for $n=2$; the papers [Hi] and [A] by Hirschowitz and Alexander, respectively, cover most of the known results for $n \geq 3$.

In this paper, by using elementary linear algebra, we give an upper bound for the regularity index of $A$ when the points are in general position (see Theorem

Received by the editors February 11, 1991 and, in revised form, November 6, 1991.

1991 Mathematics Subject Classification. Primary 14M05, 14C20; Secondary 13H15, 13 D40.

Key words and phrases. Regularity index, points in general position, rational normal curve.

The first and third authors were partially supported by M.P.I. (Italy). 
6). This bound is attained for points lying on a rational normal curve (see Proposition 7). When $n=2$ we get the same bound found in [C1] (see also [CG, Theorem 3.3]). Our approach is purely algebraic but in the last part of the paper we also give a geometric interpretation of the proofs.

\section{MAIN RESULT} 5.

In this section we state and prove the main result of the paper, Proposition

Many of the proofs require induction arguments on the number of points being considered. We begin by finding the index of regularity of the ring $A=k\left[X_{0}, \ldots, X_{n}\right] / \wp^{a}$, where (with no loss of generality) we assume $\wp=$ $\left(X_{1}, \ldots, X_{n}\right)$ corresponds to the point $P=(1,0, \ldots, 0)$. Since $X_{0} \notin \wp$ we can use our observation above on the ring $B=A / X_{0} A$ to see that $r(A)=a-1$.

Lemma 1. Let $P_{1}, \ldots, P_{r}, P$ be distinct points in $\mathbf{P}_{k}^{n}$ and let $\wp$ be the defining prime ideal of $P$. If $m_{1}, \ldots, m_{r}$, and a are positive integers, $J:=\wp_{1}^{m_{1}} \cap \wp_{2}^{m_{1}} \cap$ $\cdots \cap \wp_{r}^{m_{r}}$, and $I:=J \cap \wp^{a}$, then

$$
r(R / I)=\max \left\{a-1, r(R / J), r\left(R /\left(J+\wp^{a}\right)\right)\right\} .
$$

Proof. From the exact sequence of vector spaces

$$
0 \rightarrow I_{t} \rightarrow J_{t} \oplus\left(\wp^{a}\right)_{t} \rightarrow\left(J+\wp^{a}\right)_{t} \rightarrow 0
$$

it is clear that

$$
H_{R / I}(t)=H_{R / \wp^{a}}(t)+H_{R / J}(t)-H_{R /\left(J+\wp^{a}\right)}(t)
$$

for every integer $t$. Since the Hilbert functions of the one-dimensional CohenMacaulay rings $R / I, R / \wp^{a}$, and $R / J$ are strictly increasing until they reach the multiplicity of the ring in question and since $R /\left(J+\wp^{a}\right)$ is artinian, we see that $H_{R / I}(t)=e(R / I)$ if and only if $H_{R / \wp^{a}}(t)=a-1=e\left(R / \wp^{a}\right), H_{R / J}(t)=e(R / J)$, and $H_{R /\left(J+\wp^{a}\right)}(t)=0$. The conclusion follows.

As a consequence of the above result we get the following lower bound for the regularity index of any zero-cycle in $\mathbf{P}_{k}^{n}$.

Corollary 2. Let $s \geq 2, P_{1}, \ldots, P_{s}$ be distinct points in $\mathbf{P}_{k}^{n}$, and $m_{1} \geq m_{2} \geq$ $\cdots \geq m_{s}$ be positive integers. If $I:=\wp_{1}^{m_{1}} \cap \wp_{2}^{m_{2}} \cap \cdots \cap \wp_{s}^{m_{s}}$ then $r(R / I) \geq$ $m_{1}+m_{2}-1$.

Proof. We may assume $\wp_{1}=\left(X_{0}, X_{2}, \ldots, X_{n}\right)$ and $\wp_{2}=\left(X_{1}, \ldots, X_{n}\right)$, and we let $J=\wp_{1}^{m_{1}} \cap \wp_{3}^{m_{3}} \cap \cdots \cap \wp_{s}^{m_{s}}$. Then it is clear that $X_{0}^{m_{1}-1} X_{1}^{m_{2}-1} \notin \wp_{1}^{m_{1}}+\wp_{2}^{m_{2}}$. Since $J+\wp_{2}^{m_{2}} \subseteq \wp_{1}^{m_{1}}+\wp_{2}^{m_{2}}$, it follows that $X_{0}^{m_{1}-1} X_{1}^{m_{2}-1} \notin J+\wp_{2}^{m_{2}}$. This proves that $r\left(R /\left(J+\wp_{2}^{m_{2}}\right)\right) \geq m_{1}+m_{2}-1$ and the conclusion follows by the above lemma.

Remark. If we fix the exponents $m_{1}, \ldots, m_{s}$, then the best lower bound for $r(R / I)$ is given by the regularity index of $s$ generic fat points with those exponents. This bound is difficult to compute (see the papers [Hi, A] for the special case $m_{1}=\cdots=m_{s}=2$ ).

If we want to use the formula given in Lemma 1 then we need to find a good bound for the regularity index of the graded ring $R /\left(J+\wp^{a}\right)$. In the following lemma we give some basic properties of this artinian ring. 
Lemma 3. Let $P_{1}, \ldots, P_{r}, P$ be distinct points in $\mathbf{P}_{k}^{n}$ and $\wp$ the defining prime ideal of $P$. If $m_{1}, \ldots, m_{r}, a$ are positive integers and $J:=\wp_{1}^{m_{1}} \cap \wp_{2}^{m_{2}} \cap \cdots \cap \wp_{r}^{m_{r}}$, then

(a) $H_{R /\left(J+\wp^{a}\right)}(t)=\sum_{i=0}^{a-1} \operatorname{dim}_{k}\left[\left(J+\wp^{i}\right) /\left(J+\wp^{i+1}\right)\right]_{t}$ for every $t \geq 0$.

(b) If $P=(1,0, \ldots, 0)$ then $\left[\left(J+\wp^{i}\right) /\left(J+\wp^{i+1}\right)\right]_{t}=0$ if and only if $i>t$ or $X_{0}^{t-i} M \in J+\wp^{i+1}$ for every monomial $M$ of degree $i$ in $X_{1}, \ldots, X_{n}$.

Proof. The first assertion follows by using the exact sequences

$$
0 \rightarrow\left(J+\wp^{i}\right) /\left(J+\wp^{i+1}\right) \rightarrow R /\left(J+\wp^{i+1}\right) \rightarrow R /\left(J+\wp^{i}\right) \rightarrow 0
$$

for $i=1, \ldots, a-1$. As for (b) it follows easily from the assumption $P=$ $(1,0, \ldots, 0)$ which implies $\wp=\left(X_{1}, \ldots, X_{n}\right)$.

Now we need the following result, which has a combinatorial flavour. Recall that a set of points in $\mathbf{P}_{k}^{n}$ is said to be in general position if no $h+2$ of them are on an $h$-plane for $h<n$.

Lemma 4. Let $P_{1}, \ldots, P_{r}, P$ be distinct points in general position in $P_{k}^{n}$, let $m_{1} \geq \cdots \geq m_{r}$ be positive integers, and let $J:=\wp_{1}^{m_{1}} \cap \wp_{2}^{m_{2}} \cap \cdots \cap \wp_{r}^{m_{r}}$. If $t$ is an integer such that $n t \geq \sum m_{i}$ and $t \geq m_{1}$, we can find $t$ hyperplanes, say $L_{1}, \ldots, L_{t}$, avoiding $P$ such that $L_{1} \cdots L_{t} \in J$.

Proof. If $r \leq n$, by the general position assumption we can find a hyperplane $L$ avoiding $P$ and passing through $P_{1}, \ldots, P_{r}$. Since $t \geq m_{1}$, we have

$$
L^{t} \in \wp_{1}^{t} \cap \wp_{2}^{t} \cap \cdots \cap \wp_{r}^{t} \subseteq J .
$$

Hence we get the conclusion if $r \leq n$ and, in particular, if $\sum m_{i} \leq n$. Thus we may assume $r \geq n+1$ and argue by induction on $\sum m_{i}$. By the general position assumption we may find a hyperplane, say $L$, avoiding $P$ and passing through $P_{1}, \ldots, P_{n}$. Since $n t \geq \sum m_{i}$, we have

$$
n(t-1) \geq \sum m_{i}-n=\left(m_{1}-1\right)+\cdots+\left(m_{n}-1\right)+m_{n+1}+\cdots+m_{r} .
$$

On the other hand, since $t \geq m_{1}$ and $n t \geq \sum m_{i} \geq(n+1) m_{n+1}$, it follows that

$$
t-1 \geq\left\{m_{1}-1, \ldots, m_{n}-1, m_{n+1}, \ldots, m_{r}\right\} .
$$

Thus, by the inductive assumption we can find $t-1$ hyperplanes, say $L_{2}, \ldots, L_{t}$ avoiding $P$ and such that

$$
L_{2} \cdots L_{t} \in \wp_{1}^{m_{1}-1} \cap \cdots \cap \wp_{n}^{m_{n}-1} \cap \wp_{n+1}^{m_{n+1}} \cap \cdots \cap \wp_{r}^{m_{r}} .
$$

This implies $L L_{2} \cdots L_{t} \in J$ and the conclusion follows.

Now we come to the main result of this paper.

Proposition 5. Let $P_{1}, \ldots, P_{r}, P$ be distinct points in general position in $\mathbf{P}_{k}^{n}$ and $\wp$ the defining prime ideal of $P$. Further let $m_{1} \geq \cdots \geq m_{r} \geq a$ be positive integers, $J:=\wp_{1}^{m_{1}} \cap \wp_{2}^{m_{2}} \cap \cdots \cap \wp_{r}^{m_{r}}, m:=\sum m_{i}$, and $t$ the least integer such that $n t \geq m+a-1$. Then

$$
r\left(R /\left(J+\wp^{a}\right)\right) \leq \max \left\{m_{1}+a-1, t\right\} .
$$

Proof. Let us assume $P=(1,0, \ldots, 0)$ and so $\wp=\left(X_{1}, \ldots, X_{n}\right)$. If $r \leq n$ we can find a hyperplane, say $L$, such that $L$ contains $P_{1}, \ldots, P_{r}$ and does not contain $P$. Then, by scaling if necessary, $L=X_{0}+H$ for some linear form 
$H \in \wp$. Since it is clear that $L^{m_{1}} \in J$, we get $X_{0}^{m_{1}} \in J+\wp$. Let $i$ be any integer $0 \leq i \leq a-1$, and let $M$ be a monomial of degree $i$ in $X_{1}, \ldots, X_{n}$. Then $M \in \wp^{i}$, hence $X_{0}^{m_{1}} M \in J+\wp^{i+1}$, which implies $X_{0}^{m_{1}+a-1-i} M \in J+\wp^{i+1}$. By Lemma 3 we get $r\left(R /\left(J+\wp^{a}\right)\right) \leq m_{1}+a-1$, as desired.

Now let $r \geq n$; after a suitable change of coordinates we may further assume that $P_{1}=(0,1,0, \ldots, 0), \ldots, P_{n}=(0,0, \ldots, 1)$.

Let $h=\max \left\{m_{1}+a-1, t\right\}$. By Lemma 3(a) we need to prove that

$$
\left[\left(J+\wp^{i}\right) /\left(J+\wp^{i+1}\right)\right]_{h}=0
$$

for every $i=0, \ldots, a-1$. Since $h>i$, by Lemma $3(\mathrm{~b})$ this is equivalent to proving that

$$
X_{0}^{h-i} M \in J+\wp^{i+1}
$$

for every $i=0, \ldots, a-1$ and every monomial $M$ of degree $i$ in $X_{1}, \ldots, X_{n}$. Let $M+X_{1}^{c_{1}} \cdots X_{n}^{c_{n}}$ with $\sum c_{k}=i$. Then $m_{j}+c_{j} \geq m_{j} \geq a>a-1 \geq i$, hence $m_{j}-i+c_{j}>0$.

On the other hand, for every $j=1, \ldots, n$, we have $c_{j} \leq i$, hence $m_{j}-i+$ $c_{j} \leq m_{j}$. Since $h \geq m_{1}+a-1$ and $i \leq a-1$, this implies

$$
h-i \geq m_{1} \geq \max \left\{m_{1}-i+c_{1}, \ldots, m_{n}-i+c_{n}, m_{n+1}, \ldots, m_{r}\right\} .
$$

Moreover, $h \geq t$, hence $n h \geq m+a-1$. This implies

$$
n(h-i) \geq m+a-1-n i \geq m+i-n i=\sum_{j=1}^{n}\left(m_{j}-i+c_{j}\right)+\sum_{j=n+1}^{r} m_{j} .
$$

Thus we may use Lemma 4 to find $h-i$ hyperplanes, say $F_{1}, \ldots, F_{h-i}$, avoiding $P$ and such that

$$
F_{1} \cdots F_{h-i} \in \wp_{1}^{m_{1}-i+c_{1}} \cap \cdots \cap \wp_{n}^{m_{n}-i+c_{n}} \cap \wp_{n+1}^{m_{n+1}} \cap \cdots \cap \wp_{r}^{m_{r}} .
$$

Since it is clear that

$$
M=X_{1}^{c_{1}} \cdots X_{n}^{c_{n}} \in \wp_{1}^{i-c_{1}} \cap \cdots \cap \wp_{n}^{i-c_{n}},
$$

we get $M F_{1} \cdots F_{h-i} \in J$.

But the hyperplanes $F_{j}$ do not contain $P$, hence for every $j=1, \ldots, h-$ $i$ we can write $F_{j}=X_{0}+G_{j}$ for suitable linear forms $G_{j} \in \wp$. We get $M\left(X_{0}+G_{1}\right) \cdots\left(X_{0}+G_{h-i}\right) \in J$ and since $M G_{j} \in \wp^{i+1}$ for every $j$, this implies $X_{0}^{h-i} M \in J+\wp^{i+1}$ as wanted.

From now on if $\alpha$ is a rational number, denote by $[\alpha]$ its integer part. With this notation it is clear that for positive integers $q$ and $n$ we have

$$
q+n>n[(q+n-1) / n] \geq q .
$$

This implies

$$
[(q+n-1) / n]=\min \{t \mid t n \geq q\} .
$$

Theorem 6. Let $s \geq 2, P_{1}, \ldots, P_{s}$ be distinct points in general position in $\mathbf{P}_{k}^{n}$, and $m_{1} \geq \cdots \geq m_{s}$ be positive integers. Further let $I=\wp_{1}^{m_{1}} \cap \cdots \cap \wp_{s}^{m_{s}}$. Then

$$
r(R / I) \leq \max \left\{m_{1}+m_{2}-1,\left[\left(\sum m_{i}+n-2\right) / n\right]\right\} .
$$

Proof. Let $J:=\wp_{1}^{m_{1}} \cap \cdots \cap \wp_{s-1}^{m_{s-1}}$. By Lemma 1 we have

$$
r(R / I)=\max \left\{m_{s}-1, r(R / J), r\left(R /\left(J+\wp_{s}^{m_{s}}\right)\right)\right\} .
$$


Let $s=2$. Since $m_{1}+m_{2}-1 \geq \min \left\{t \mid n t \geq m_{1}+m_{2}-1\right\}$, we get

$$
r\left(R /\left(J+\wp_{2}^{m_{2}}\right)\right) \leq m_{1}+m_{2}-1
$$

by Proposition 5. Note that $r(R / J)=r\left(R / \wp_{1}^{m_{1}}\right)=m_{1}-1$. Then $r(R / I) \leq$ $m_{1}+m_{2}-1$ and the conclusion follows in this case. Thus we may argue by induction on $s$. By the inductive assumption we have

$$
r(R / J) \leq \max \left\{m_{1}+m_{2}-1,\left[\left(\sum_{i=1}^{s-1} m_{i}+n-2\right) / n\right]\right\},
$$

and by the above proposition

$$
r\left(R /\left(J+\wp_{s}^{m_{s}}\right)\right) \leq \max \left\{m_{1}+m_{s}-1,\left[\left(\sum_{i=1}^{s} m_{i}+n-2\right) / n\right]\right\} .
$$

Hence the conclusion is immediate.

We prove now that the bound found in Theorem 6 is sharp for points lying on a rational normal curve.

Proposition 7. Let $s \geq 2, P_{1}, \ldots, P_{s}$ be distinct points on a rational normal curve in $\mathbf{P}_{k}^{n}$, and $m_{1} \geq \cdots \geq m_{s}$ be positive integers. Further let $I=\wp_{1}^{m_{1}} \cap \cdots \cap$ $\wp_{s}^{m_{s}}$. Then

$$
r(R / I)=\max \left\{m_{1}+m_{2}-1,\left[\left(\sum m_{i}+n-2\right) / n\right]\right\} .
$$

Proof. We recall that all rational normal curves in $\mathbf{P}^{n}$ are isomorphic under a linear change of coordinates. Hence, without loss of generality, we may assume that the points are on the curve with parametric equations

$$
X_{0}=t^{n}, X_{1}=t^{n-1} u, \ldots, X_{n-1}=t u^{n-1}, X_{n}=u^{n} \text {. }
$$

Also it is clear that a rational normal curve in $\mathbf{P}^{n}$ is a nondegenerate curve of degree $n$, which implies that the points are in general position. Put $t:=$ [( $\left.\left.\sum m_{i}+n-2\right) / n\right]$. If $t \leq m_{1}+m_{2}-1$, the conclusion follows by Corollary 2 and Theorem 6. Hence we may assume that $t \geq m_{1}+m_{2}$ and, as usual, that $\wp_{s}=\left(X_{1}, \ldots, X_{n}\right)$. Further we let $J=\wp_{1}^{m_{1}} \cap \wp_{2}^{m_{2}} \cap \cdots \cap \wp_{s-1}^{m_{s-1}}$. We claim that

$$
X_{0}^{(t-1)-\left(m_{s}-1\right)} X_{1}^{m_{s}-1} \notin J+\wp_{s}^{m_{s}} .
$$

In fact, if $X_{0}^{(t-1)-\left(m_{s}-1\right)} X_{1}^{m_{s}-1} \in J+\wp_{s}^{m_{s}}$, then for some $F \in\left[\wp_{s}^{m_{s}}\right]_{t-1} \subseteq \wp_{s}^{m_{s}-1}$ we have $H:=X_{0}^{(t-1)-\left(m_{s}-1\right)} X_{1}^{m_{s}-1}+F \in J$. Since $X_{0}^{(t-1)-\left(m_{s}-1\right)} X_{1}^{m_{s}-1} \in \wp_{s}^{m_{s}-1}$, we get $H \in J \cap \wp_{s}^{m_{s}-1}$. By the definition of $t$ and the remark before Theorem 6 , we have $\sum m_{i}-1>n(t-1)$, hence by Bezout's theorem we get that the hypersurface corresponding to $H$ contains the rational curve $C$ on which are our points. This implies that $H$ must vanish on the point $\left(1, \alpha, \alpha^{2}, \ldots, \alpha^{n}\right)$ for every $\alpha \in k$ and thus that $\alpha^{m_{s}-1}+F\left(1, \alpha, \alpha^{2}, \ldots, \alpha^{n}\right)=0$ for every $\alpha \in k$.

Since $F \in \wp_{s}^{m_{s}}$, we have $F\left(1, \alpha, \alpha^{2}, \ldots, \alpha^{n}\right)=\alpha^{m_{s}} G(\alpha)$ for some polynomial $G \in k[X]$, hence we get $\alpha^{m_{s}-1}+\alpha^{m_{s}} G(\alpha)=0$ for every $\alpha \in k$, a contradiction. This proves the claim. But then

$$
X_{0}^{(t-1)-\left(m_{s}-1\right)} X_{1}^{m_{s}-1} \notin J+\wp_{s}^{m_{s}} .
$$


This implies $r\left(R /\left(J+\wp_{s}^{m_{s}}\right)\right) \geq t$. By Lemma 1 we get $r(R / I) \geq t$ and the conclusion follows from the above theorem.

Remark. The above bound for the regularity index can be attained also by fat points not lying on a rational normal curve (see $[\mathrm{Cl}, \S 6]$ ).

We end this section with the following result, which deals with the extremal case $r(R / I)=m_{1}+m_{2}-1$ (see Corollary 2).

Corollary 8. Let $n \geq 3,2 \leq s \leq n+2$, and let $P_{1}, \ldots, P_{s}$ be distinct points in general position in $\mathbf{P}_{k}^{n}$. If $2 \leq m_{1} \geq m_{2} \geq \cdots \geq m_{s}>0$ are given integers and $I=\wp_{1}^{m_{1}} \cap \cdots \cap \wp_{s}^{m_{s}}$, then $r(R / I)=m_{1}+m_{2}-1$.

Proof. By Corollary 2 and Theorem 6 we need only prove that $n\left(m_{1}+m_{2}-1\right) \geq$ $\sum m_{i}-1$ or, equivalently,

$$
\left(m_{1}+m_{2}-1\right)(n-1)-\left(m_{3}+\cdots+m_{s}\right) \geq 0 .
$$

But we have

$$
\begin{aligned}
\left(m_{1}\right. & \left.+m_{2}-1\right)(n-1)-\left(m_{3}+\cdots+m_{s}\right) \\
& \geq\left(m_{1}+m_{2}-1\right)(n-1)-n m_{2}=\left(m_{1}-1\right)(n-1)-m_{2} \\
& \geq\left(m_{1}-1\right)(n-1)-m_{1}=\left(m_{1}-1\right)(n-2)-1 .
\end{aligned}
$$

The conclusion follows from the assumptions $n \geq 3$ and $m_{1} \geq 2$.

\section{A GEOMETRIC INTERPRETATION}

We recall that if $Z$ is the zero-dimensional subscheme of $\mathbf{P}_{k}^{n}$ corresponding to the ideal $I=\wp_{1}^{m_{1}} \cap \cdots \cap \wp_{s}^{m_{s}}$, then for every integer $t$ we can consider the linear system $\mathscr{L}_{t}$ of all hypersurfaces of degree $t$ containing $Z$ as a subscheme. It is clear that

$$
\begin{aligned}
\operatorname{dim}_{k}\left(\mathscr{L}_{t}\right) & =\operatorname{dim}_{k}\left(I_{t}\right)-1=\operatorname{dim}_{k}\left(R_{t}\right)-H_{R / I}(t)-1 \\
& =\operatorname{dim}_{k}\left(R_{t}\right)-e+h\left(\mathscr{L}_{t}\right)-1,
\end{aligned}
$$

where $h\left(\mathscr{L}_{t}\right):=e-H_{R / I}(t)$ is called the superabundance of $\mathscr{L}_{t}$. The linear system $\mathscr{L}_{t}$ is said to be regular iff $h\left(\mathscr{L}_{t}\right)=0$, that is, $H_{R / I}(t)=e$ or, with the notation of the above sections, $r(R / I) \leq t$.

We fix the following notation: $J:=\wp_{1}^{m_{1}} \cap \cdots \cap \wp_{s}^{m_{s}}, I:=J \cap \wp^{a}, I^{\prime}:=$ $J \cap \wp^{a-1}, e=e(R / I), e^{\prime}=e\left(R / I^{\prime}\right)$. It is clear that

$$
\begin{aligned}
e & =\sum_{i=1}^{s}\left(\begin{array}{c}
m_{i}+n-1 \\
n
\end{array}\right)+\left(\begin{array}{c}
a+n-1 \\
n
\end{array}\right), \\
e^{\prime} & =\sum_{i=1}^{s}\left(\begin{array}{c}
m_{i}+n-1 \\
n
\end{array}\right)+\left(\begin{array}{c}
a+n-2 \\
n
\end{array}\right),
\end{aligned}
$$

hence

$$
e-e^{\prime}=\left(\begin{array}{c}
a+n-2 \\
n-1
\end{array}\right) .
$$

By using the formula for the dimension of the sum of two vector spaces in terms of the dimensions of the summands and that of the intersection, we have

$$
\begin{aligned}
\operatorname{dim}_{k}\left(I_{t}^{\prime}\right)-\operatorname{dim}_{k}\left(I_{t}\right) & =\operatorname{dim}\left(\wp^{a-1} / \wp^{a}\right)_{t}-\operatorname{dim}\left(\left(J+\wp^{a-1}\right) /\left(J+\wp^{a}\right)\right)_{t} \\
& \leq \operatorname{dim}\left(\wp^{a-1} / \wp^{a}\right)_{t} \leq e-e^{\prime}
\end{aligned}
$$


Lemma 9. The linear system $\mathscr{L}_{t}$ is regular if and only if the linear system $\mathscr{L}_{t}^{\prime}$ is regular and $\operatorname{dim}_{k}\left(I^{\prime} / I\right)_{t} \geq e-e^{\prime}$.

Proof. The conclusion follows immediately from the above remark and the exact sequence $0 \rightarrow I^{\prime} / I \rightarrow R / I \rightarrow R / I^{\prime} \rightarrow 0$.

Now it is clear that in order to prove Theorem 6 using induction on the sum of the multiplicities, we need only prove that there exist $e-e^{\prime}$ forms in $I_{t}^{\prime}$ that are $k$-linearly independent modulo $I_{t}$. This can be proved as in Proposition 5 .

This approach has a nice geometric interpretation. We shall see that the existence of such $e-e^{\prime}$ forms is equivalent to the fact that the hypersurfaces of $\mathscr{L}_{t}^{\prime}$ separate the directions at the fat point $P$. To explain this fact we need some more notation.

Let $\pi$ be the hyperplane $X_{0}=0$. We may consider $\pi$ as $\mathbf{P}_{k}^{n-1}$ with coordinate ring $S=k\left[X_{1}, \ldots, X_{n}\right]$. Since

$$
\operatorname{dim}_{k}\left(S_{a-1}\right)=\left(\begin{array}{c}
n+a-2 \\
a-1
\end{array}\right)=e-e^{\prime},
$$

we may find $e-e^{\prime}$ points in $\mathbf{P}_{k}^{n-1}$, say $Q_{1}, \ldots, Q_{e-e^{\prime}}$, that are not on a hypersurface of degree $a-1$ in $\mathbf{P}_{k}^{n-1}$. This implies that for every $i=1, \ldots, e-$ $e^{\prime}$, there is a form $G_{i} \in S_{a-1}$ such that $G_{i}\left(Q_{i}\right) \neq 0$ and $G_{i}\left(Q_{j}\right)=0$ if $j \neq i$. We consider for every $i=1, \ldots, e-e^{\prime}$ the line $L_{i}$ connecting $P$ and $Q_{i}$.

With this notation we say that the hypersurfaces of $\mathscr{L}_{t}^{\prime}$ separate the directions at the fat point $P$ if there exist hypersurfaces $F_{1}, \ldots, F_{e-e^{\prime}}$ in $\mathscr{L}_{t}^{\prime}$ such that each $F_{i}$ contains the subscheme $a P$ of $L_{j}$ for every $j \neq i$ and does not contain the subscheme $a P$ of $L_{i}$.

Proposition 10. There exist $e-e^{\prime}$ elements in $I_{t}^{\prime}$ that are $k$-linearly independent modulo $I_{t}$ if and only if the hypersurfaces of $\mathscr{L}_{t}^{\prime}$ separate the directions at the fat point $P$.

Proof. Let $F_{1}, \ldots, F_{e-e^{\prime}}$ be the elements of $I_{t}^{\prime}$ that are $k$-linearly independent modulo $I_{t}$. Since $\operatorname{dim}_{k}\left(\wp^{a-1} / \wp^{a}\right)_{t}=e-e^{\prime}$ and $F_{1}, \ldots, F_{e-e^{\prime}}$ are $k$-linearly independent also modulo $\wp_{t}^{a}$, they form a basis for the vector space $\left(\wp^{a-1} / \wp^{a}\right)_{t}$. The elements $G_{1}, \ldots, G_{e-e^{\prime}}$ defined above can be considered as elements of $\wp^{a-1}$. Hence for every $i=1, \ldots, e-e^{\prime}$ we can write

$$
X_{0}^{t-(a-1)} G_{i}=\sum \lambda_{i j} F_{j}+H_{i}
$$

for suitable $\lambda_{j} \in k$ and $H_{i} \in \wp^{a}$. Due to the choice of $G_{1}, \ldots, G_{e-e^{\prime}}$ it is easy to see that for $i$ running from 1 to $e-e^{\prime}$, the hypersurfaces corresponding to $\sum \lambda_{i j} F_{j}$ separate the directions at the fat point $P$.

Conversely, let $F_{1}, \ldots, F_{e-e^{\prime}}$ be the elements of $I_{t}^{\prime}$ given by our assumption. If $\sum \lambda_{i} F_{i} \in I_{t}$, then $\sum \lambda_{i} F_{i} \in \wp^{a}$. It follows that $\sum \lambda_{i} F_{i}$ contains the subscheme $a P$ of $L_{i}$ for every $i$. This clearly implies $\lambda_{i}=0$ for every $i$, and we are done.

\section{REFERENCES}

[A] J. Alexander, Singularités imposables en position général à une hypersurface projective, Compositio Math. 68 (1988), 305-354.

[C] G. Castelnuovo, Ricerche generali sopra i sistemi lineari di curve piane, Mem. Reale Accad. Sci. Torino 42 (1891), 137-188. 
[C1] M. V. Catalisano, Linear systems of lane curves through fixed "fat" points of $\mathbf{P}^{2}$, J. Algebra 142 (1991), 81-100.

[C2] _ "Fat" points on a conic, Comm. Algebra 19 (1991), 2153-2168.

[CG] M. V. Catalisano and S. Greco, Linear systems: developments of some results by $F$. Enriques and B. Segre, Lecture Notes in Pure and Appl. Math., vol. 132, Dekker, New York, 1991, pp. 41-57.

[DG] E. Davis and A. Geramita, The Hilbert Function of a special class of 1-dimensional CohenMacaulay graded algebras, The Curves Seminar at Queen's, Queen's Papers in Pure and Appl. Math. 67 (1984), 1H-29H.

[G1] A. Gimigliano, Regularity of linear systems of plane curves, J. Algebra 124 (1989), 447-460.

[G2] _ Our thin knowledge on fat points, The Curve Seminar at Queen's III, Queen's Papers in Pure and Appl. Math. 83 (1989), 1B-50B.

[G] S. Greco, Remarks on the postulation of zero-dimensional subschemes of projective space, Math. Ann. 284 (1989), 343-351.

[H] B. Harbourne, Complete linear systems on rational surfaces, Trans. Amer. Math. Soc. 289 (1985), 213-226.

[Hi] A. Hirschowitz, La méthod d'Horace pour l'Interpolation á plusieurs variables, Manuscripta Math. 50 (1985), 337-388.

[S] B. Segre, Alcune questioni su insiemi finit di punit in Geometria Algebrica, Atti Convegno Internaz. Geometria Algebrica, Torino, 1961, pp. 15-33.

(N. V. Trung) Institute of Mathematics, Box 631, Bó Hô, Hanoi, Vietnam

(M. V. Catalisano and G. Valla) Dipartimento di Matematica, Università di Genova, Via L. B. Alberti 4, 16132 Genova, Italy

E-mail address, M. V. Catalisano: arezzo@igecuniv.bitnet

E-mail address, G. Valla: valla@igecuniv.bitnet 